\title{
The Immune-Regulatory Effect of the Exosomes from Human Umbilical Cord Blood Plasma on T Cells in vitro
}

\author{
Weiyi Xia ${ }^{1}$, Zheng Ye ${ }^{1}$, Hailing $\mathrm{Li}^{2}$, Doulathunnisa Jaffar Ali ${ }^{1}$, Jiahua Ding ${ }^{3}$, Bo Sun ${ }^{1}$, \\ Zhongdang Xiao ${ }^{1}$ \\ ${ }^{1}$ State Key Laboratory of Bioelectronics, School of Biological Science and Medical Engineering, Southeast University, Nanjing, China \\ ${ }^{2}$ Zhongda Hospital, Department of Obstetrics \& Gynecology,Southeast University, Nanjing, China \\ ${ }^{3}$ Zhongda Hospital, Department of Hematology, Key Deptment Jiangsu Medicine, Southeast University, Nanjing, China
}

\section{Email address:}

zdxiao@seu.edu.cn (Zhongdang Xiao)

\section{To cite this article:}

Weiyi Xia, Zheng Ye, Hailing Li, Doulathunnisa Jaffar Ali, Jiahua Ding, Bo Sun, Zhongdang Xiao. The Immune-Regulatory Effect of the Exosomes from Human Umbilical Cord Blood Plasma on T Cells in vitro. Science Discovery. Vol. 6, No. 1, 2018, pp. 35-42.

doi: $10.11648 /$ j.sd.20180601.16

Received: January 19, 2018; Accepted: February 22, 2018; Published: May 23, 2018

\begin{abstract}
To investigate the immune-regulatory function of exosomes from human umbilical cord blood plasma on T cells in peripheral blood, differential centrifugation was used to isolate the exosomes from umbilical cord blood plasma (Pexo) firstly. The exosome was characterized by transmission electronic microscope and the western blot of CD63, Lamp2. Exosomes were cultured with peripheral blood lymphocytes which were isolated from the peripheral blood for $72 \mathrm{~h}$, and analyzed for the changes of the Treg subpopulation and memory $\mathrm{T}$ cells by flow cytometry, and their effect on lymphocyte proliferation was measured by CFSE assay. The production of IFN- $\gamma$, IL-2, TNF- $\alpha$, TGF- $\beta$ was detected by qRT-PCR. The results show that Pexo could not activate $\mathrm{T}$ cells or proliferate the $\mathrm{T}$ cells in vitro, and could not regulate the Treg subpopulation or memory $\mathrm{T}$ cells, but the expression of IFN- $\gamma$ and TNF- $\alpha$ was decreased significantly after the treatment with Pexo within $24 \mathrm{~h}$. The exosomes from the umbilical cord blood plasma can decrease the expression of inflammatory factors observably under the condition of keeping the activity and function of the cells. So it has the potential to be used to cure the related immunological diseases due to the inflammatory factors overexpression, especially for the elderly, as their body are usually under the inflammatory state.
\end{abstract}

Keywords: Human Umbilical Cord Blood Plasma, Immune, Exosome, Treg Cells

\section{脐血浆外泌体对 $\mathbf{T}$ 细胞调节作用研究}

\author{
夏威夷 ${ }^{1}$, 叶正 ${ }^{1}$, 李海玲 ${ }^{2}$, Doulathunnisa $\mathrm{Jaffar}_{\mathrm{Ali}^{1}}$, 丁家华 ${ }^{3}$, 孙博 ${ }^{1}$, 肖忠党 ${ }^{1}$ \\ ${ }^{1}$ 东南大学生物电子学国家重点实验室, 东南大学生物医学工程学院, 南京, 中国 \\ 2东南大学附属中大医院妇产科, 南京, 中国 \\ 3 东南大学附属中大医院血液科, 南京, 中国
}

邮箱

zdxiao@seu.edu.cn (肖忠党)

摘要：为了探讨人脐血浆外泌体对外周血中 $\mathrm{T}$ 细胞的免疫调节作用，首先用差速离心法提取脐血浆外泌体（Pexo）。 通过TEM和Western Blot表征鉴定外泌体; 将外泌体与人外周血单个核细胞(PBMC)共培养 $72 \mathrm{~h}$, 通过流式检测作用前后 调节性 T细胞 (Treg) 和记忆性T细胞的变化, 用CFSE染色检Pexo对PBMCs增殖的影响, qRT-PCR检测IFN- $\gamma 、$ IL-2、 TNF- $\alpha$ 、 TGF- $\beta$ 的水平变化。结果表明 Pexo 不能在体外激活 $\mathrm{T}$ 细胞或者促进 $\mathrm{T}$ 细胞增殖, 也不能调节 $\mathrm{CD}^{+} \mathrm{CD} 25^{+} \mathrm{FoxP}^{+}{ }^{+}$Treg细胞亚群以及记忆性 T细胞亚群的比例, 但经Pexo处理后PBMCs分泌的IFN- $\gamma$ 以及TNF- $\alpha$ 促炎症 
因子水平在 $24 \mathrm{~h}$ 内的表达显著降低 $(\mathrm{P}<0.05)$ 。因此, 脐带血血浆的外泌体能够在不影响 $\mathrm{T}$ 淋巴细胞的活性以及各亚群功 能前体条件下, 能显著下调淋巴细胞分泌的炎症因子, 维持细胞的良好状态, 将有望用于因炎症因子过度表达的相关 免疫疾病的治疗，尤其对于老年人群因长期处于低度炎症状态下的免疫细胞活性有一个调节作用。

关键词: 脐血浆, 免疫, 外泌体, Treg细胞

\section{1. 引言}

人体的免疫系统是由胸腺、脾脏、淋巴结等免疫器官 组成的。这是人体的防御系统, 能够自动识别侵入人体的 “非已”成分，加以排斥，还能够除去受损的细胞，维持身 体的平衡。在免疫细胞中, T淋巴细胞在免疫调节中扮演 着重要的角色。 $\mathrm{T}$ 淋巴细胞根据其功能可分为 $\mathrm{Th} 1, \mathrm{Th} 2$, Th17, Treg, central memory T等细胞亚群。比如调节性 T 细胞 (Treg) 是一类可以调节其他多种免疫细胞功能的 $\mathrm{T}$ 细胞亚群, 其正常生理功能对体内免疫稳态维持必不可少 [1-5]。其功能失调与人类多种重大疾病, 如自身免疫性疾 病、感染性疾病、过敏性疾病、恶性肿瘤、移植排斥的发 生、发展及治疗都密切相关[6-8]。记忆性T淋巴细胞能在 极短的时间内清除再次进入到体内的抗原, 保护人体不再 受到同样抗原的侵袭。因此, 维持 $\mathrm{T}$ 淋巴细胞各功能亚群 的稳定对于机体的保护是最基础也是最重要的。

脐带血是胎儿娩出后残留在脐带和胎盘中的血液, 脐 血中含有丰富的活性物质和功能不成熟的免疫细胞, 这些 活性物质和免疫细胞在脐血造血干细胞的成功移植及在 抗肿瘤免疫中发挥重要作用。其中脐血浆是脐血细胞直接 接触和赖以生存的重要微环境, 含有丰富的细胞因子和其 他活性物质, 这些因子主要由脐血细胞和胎盘等其他的组 织细胞来源。脐血中的活性因子对不同脐血细胞的发育和 功能起重要的调节作用[9]。

外泌体 (exosomes) 是由各种细胞分泌的大小在 30 $100 \mathrm{~nm}$ 之间的微小双层膜囊泡, 其内含有与细胞来源相关 的miRNA、mRNA及蛋白等物质, 可以直接激活受体细胞, 也可以通过转运其包含的生物活性物质进人受体细胞内, 参与细胞间的信息交流及许多重要的生理或病理过程 [10]。

一直以来, 关于血浆外泌体研究较少, 除了从血浆中 提取外泌体较细胞培养上清复杂, 血浆里面包含了多种细 胞分泌的外泌体使得其成分复杂, 增加了对其功能研究的 难度 [11]。近年来研究表明, 血浆外泌体可以作为多种疾 病诊断的生物标志物[12], 如阿尔茨海默病[13]。也有研究 表明血浆外泌体对心肌缺血再灌注损伤也具有一定的保 护作用 [14]。最新研究表明, 不仅正常人外周血血浆里面 含有外泌体[15], 脐血浆里面也含有丰富的外泌体[16]。同 时最新研究表明, 脐血浆外泌体能够通过介导 miR-21-3p 促进血管以及成纤维细胞生产从而促进创伤的愈合 [17]。 但是目前, 只有少量文献初步探索了人外周血浆外泌体体 外对于 $\mathrm{T}$ 细胞的调节作用 [18], 而对于脐带血血浆外泌体在 体外对 $\mathrm{T}$ 淋巴细胞的研究少见报道, 因此本研究初步探索 脐血血浆外泌体对人外周血的淋巴细胞的免疫调节作用。

\section{2. 实验方法}

本实验所用的脐带血均来自于东南大学中大医院, 并 在离体 $6 \mathrm{~h}$ 内处理。

\section{1. 主要试剂}

RPMI-1640培养液、胎牛血清(FBS)(Hyclone), Ficoll (GE Healthcare), CFSE(Stemcell), 7-AAD(eBioscience), WB抗体Lamp2/CD63(Santa Cruz), 流式抗体CD4-FITC、 CD8-FITC、CD25-APC、Foxp3-PE 、CD45RA-APC、 CCR7-PE (Biolegend), RNA提取试剂盒(TakaRa), 荧光定 量试剂盒(Roche), PCR引物(金斯瑞科技有限公司)

\section{2. 脐血浆分离以及PBMCs的提取}

无菌的PBS1:1稀释全血, 将适量的Ficoll装入15ml离心 管 (Ficoll: 全血稀释液 $=1: 2$ ), 向离心管中加入全血稀释液, 使全血稀释液置于Ficoll上层, 避免混合; $1200 \mathrm{rpm}, 15^{\circ} \mathrm{C}$, 离心 $30 \mathrm{~min}$; 将离心后的上层血浆收集于4度冰箱保存待用。 人外周血操作同上，但在离心后吸取中间的白膜层至新的 $15 \mathrm{ml}$ 离心管中, $1000 \mathrm{rpm}$ 离心 $5 \mathrm{~min}$, 用PBS清洗3次, 最后用 RPMI 1640完全培养基重悬细胞, 流式细胞仪计数培养。

\section{3. 外泌体的提取}

将收集的脐血血浆先 $3000 \mathrm{~g}$, 离心 $5 \mathrm{~min}$; 接着 $8000 \mathrm{~g}$, 离心 $30 \mathrm{~min}$, 去除细胞碎片, 再将离心后的上清过 $0.22 \mu \mathrm{m}$ 滤膜, 44000rpm, 超速离心 $2 \mathrm{~h}$ 。将超速离心后的沉淀用PBS 清洗, 最后用PBS重悬分装置于-20度冰箱待用。

\section{4. 外泌体的鉴定}

将提取的外泌体样小体重悬于PBS缓冲液中, 标记磷 钨酸后滴到透射电镜碳网上。自然晾干后, 进行透射电镜 的观察, 并拍摄取图。

\section{5. 蛋白的提取}

将用PBS重悬的外泌体等体积与裂解液混合, 并加入 蛋白酶抑制剂（1m1体积加 $1 \mu 1$ 蛋白酶抑制剂），置于冰上 $10 \mathrm{~min}$, 用超声破碎仪破碎 $3 \mathrm{~min}$ 。将破碎后的蛋白收集保 存用于后续的蛋白定量。

\subsection{BCA蛋白定量及蛋白质印迹检测外泌体特异性蛋白 的表达}

将提取的蛋白用BCA法计算Pexo的浓度。30 $\mathrm{\mu g}$ / 上 样, $90 \mathrm{~V}$ 电泳 $30 \mathrm{~min}, 110 \mathrm{~V}$ 电压分离蛋白, $380 \mathrm{~mA}$ 转膜3 
$\mathrm{h}, 5 \%$ 脱脂牛奶封闭 $1 \mathrm{~h}$, 加入一抗 $4^{\circ} \mathrm{C}$ 孵育过夜, TBST洗 膜 $10 \mathrm{~min} \times 3$ 次后, 加入对应HRP标记二抗, 室温下捊育 $1 \mathrm{~h}$ 后, TBST洗膜 $10 \mathrm{~min} \times 3$ 次, 然后显色, 天能成像系统成像。

\section{7. 流式检测PBMCs 和Pexo共培养后Treg (CD4+CD25+Foxp3+) 以及记忆性 T细胞比例的变化}

将刚分离出的 $1 \times 10^{6}$ / 孔 PBMCs种植于 6 孔板, 然后将 $20 \mu \mathrm{g}$ 的Pexo加入到 6 孔板中, 同时设置空白组, 和PBMCs 共培养 72h , 收集细胞, 流式检测共培养前后 $\operatorname{Treg}\left(\mathrm{CD} 4^{+} \mathrm{CD} 25^{+} \mathrm{Foxp}^{+}\right)$以及记忆性T细胞比例的变化。

\subsection{PBMCs的CFSE染色}

用PBS稀释PBMCs, 调整细胞浓度为 $2 \times 10^{6} / \mathrm{ml}$; 每 $10^{6}$ 个细胞加入 $2 \mu \mathrm{l}, 5 \mathrm{mM} \mathrm{CFSE}, 37^{\circ} \mathrm{C}, 10 \mathrm{~min}$; 加入预冷的 含 $10 \%$ FBS的RPMI 1640培养液终止染色, 冰上 $5 \mathrm{~min}$; 再 用PBS清洗三次。

\section{9. qRT-PCR检测在加入Pexo后PBMCs 分泌的相关免疫因子}

收集反应后的细胞, 提取细胞的总mRNA, 用酶标仪 测定RNA浓度, 并立即进行反转录以获得cDNA, 然后进 行苂光定量PCR。相关基因引物序列:

IL-2 前引物 AGAACTCAAACCTCTGGAGGAAG $\left(5^{\prime}-3{ }^{\prime}\right)$

后引物GCTGTCTCATCAGCATATTCACAC (5'-3')

TGF- $\beta$ 前引物 TACCTGAACCCGTGTTGCTCTC
后引物GTTGCTGAGGTATCGCCAGGAA (5'-3') $\left(5^{\prime}-3\right.$ ')

IFN- $\gamma$ 前引物 GAGTGTGGAGACCATCAAGGAAG

后引物TGCTTTGCGTTGGACATTCAAGTC (5'-3’)

$\mathrm{TNF}-\alpha$ 前引物

CTCTTCTGCCTGCTGCACTTTG(5'-3')

后引物ATGGGCTACAGGCTTGTCACTC( $\left.5^{\prime}-3^{\prime}\right)$

$\beta$-actin前引物TCACCAACTGGGACGACAT(5'-3')

后引物GCACAGCCTGGATAGCAAC(5'-3')

\subsection{0. 统计学处理}

采用统计学软件GraphPad Prism 5.0进行分析, 数据以 mean $\pm \mathrm{SD}$ 表示, 组间比较采用 $\mathrm{t}$ 检验, $\mathrm{P}<0.05$ 为差异有统计 学意义。 $\beta$-actin前引物TCACCAACTGGGACGACAT(5'-3'), 后引物GCACAGCCTGGATAGCAAC(5'-3')

\section{3. 实验结果}

\section{1. 脐血浆外泌体（Pexo）的生物学特性}

图1是脐血浆外泌体的SEM，黑色箭头处都能较明显 看出外泌体的结构形态, 尺寸也都在 $100 \mathrm{~nm}$ 左右。因为血 浆里本身含有的成分就比较多比较复杂, 所以图像没有单 纯从细胞培养液里提取出来的外泌体干净, 但综合形态以 及尺寸，都符合外泌体的特征。 $\left(5^{\prime}-3^{\prime}\right)$

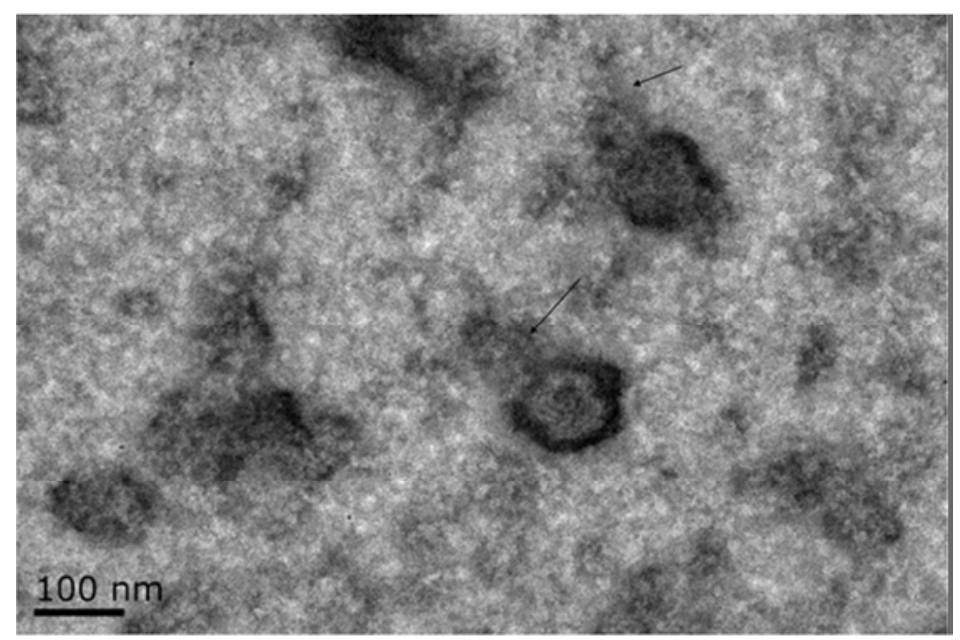

图1 脐血浆外泌体 (Pexo) 的透射电镜图。

用BCA法定量外泌体总蛋白, 测得Pexo样品蛋白浓度 约为 $1200 \mu \mathrm{g} / \mathrm{ml}$ 。蛋白质免疫印迹法检测结果显示, Pexo 表达CD63和Lamp2蛋白。

\section{2. 流式检测外周PBMCs和Pexo共培养后 Treg (CD4+CD25+Foxp3+) 以及记忆性T细胞比例的变化}

将Pexo与PBMCs共培养 $72 \mathrm{~h}$ 后, 先对调节性 $\mathrm{T}$ 细胞亚 群Treg $\left(\mathrm{CD} 4^{+} \mathrm{CD} 25^{+} \mathrm{Foxp}^{+}\right)$进行检测。与control组的Treg 亚群比例 $(6.2 \%)$ 相比, Pexo组 $(9.1 \%)$ 没有显示出明显 变化（图2)
然后是对 $\mathrm{CD} 4^{+}$记忆型 $\mathrm{T}$ 细胞的检测, 结果表明, Pexo 未对 $\mathrm{CD} 4{ }^{+}$记忆型 $\mathrm{T}$ 细胞亚群产生影响（图3）。同样地, 也未对 $\mathrm{CD}^{+}$记忆型T细胞亚群产生影响（图4）。从检测 结果上来看, 我们还发现, 在 $\mathrm{CD} 4^{+}$记忆型 $\mathrm{T}$ 细胞亚群中, $\mathrm{CD} 4^{+}$Naïve T细胞亚群几乎没有, 中央记忆型 $\mathrm{T}$ 细胞 ( TCM) 也比较少, 大多数都是效应性 $\mathrm{T}$ 细胞 ( TEM)。而在 $\mathrm{CD} 8^{+}$ 记忆型细胞亚群中, 则与 $\mathrm{CD}^{+}$细胞亚群相反, $\mathrm{CD} 8^{+}$Naïve $\mathrm{T}$ 细胞亚群和中央记忆型 $\mathrm{T}$ 细胞亚群较多, 而效应性 $\mathrm{T}$ 细胞 占比相对较少。 
A

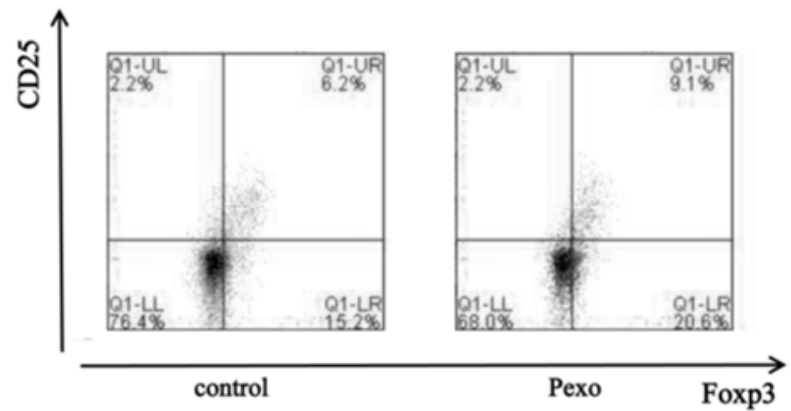

B

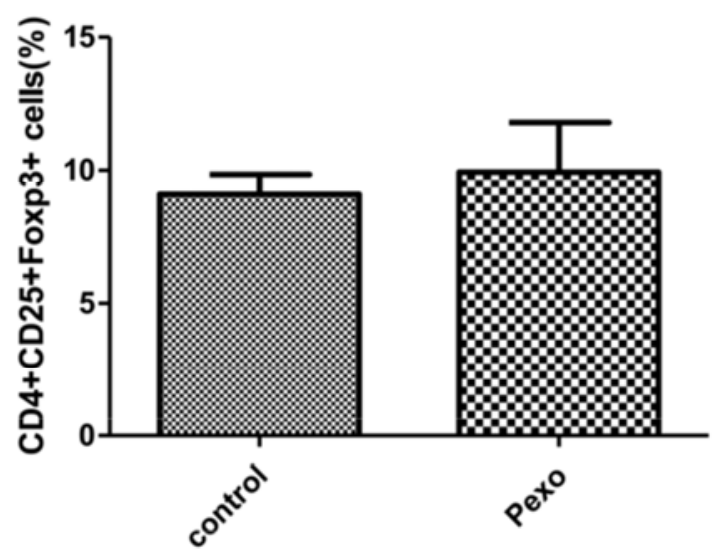

图2 Pexo对PBMCs中Treg细胞亚群的影响。

A Pexo和PBMCs共培养 $72 \mathrm{~h}$ 后检测 $T r e g$ 细胞亚群变化情况 B 对重复实 验进行统计学分析结果 $(* \mathrm{p}<0.05)$
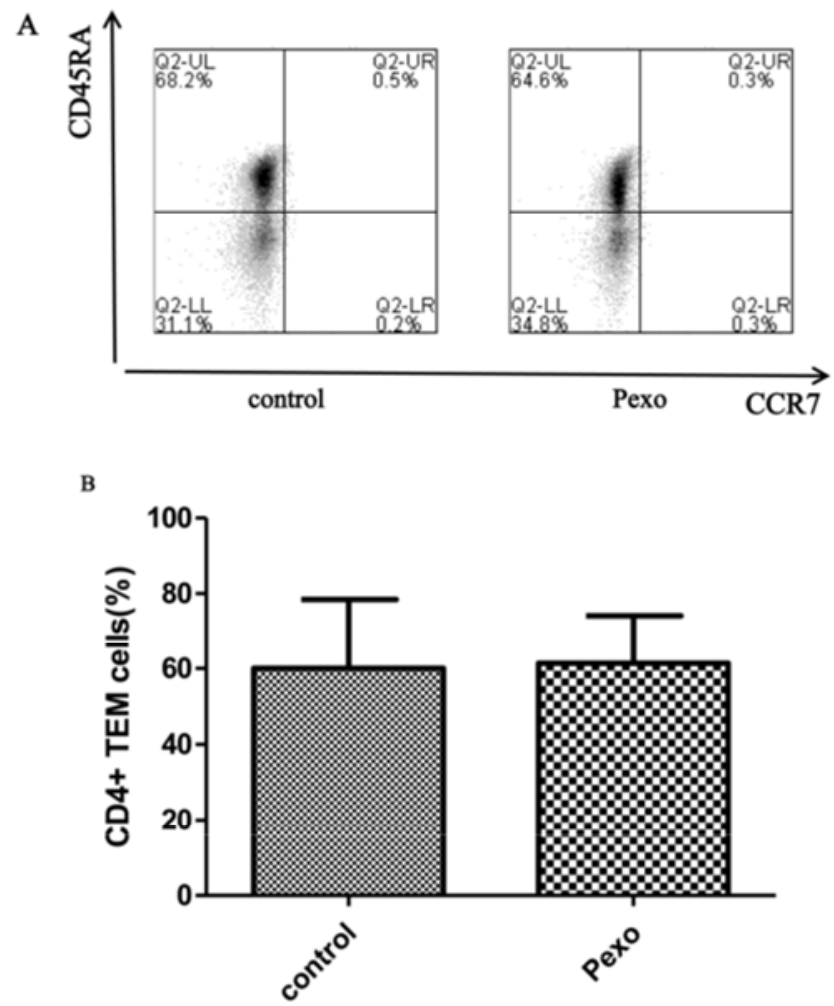

图3 Pexo对PBMCs中CD4 记忆型T细胞各亚群的影响。

A Pexo和PBMCs共培养 $72 \mathrm{~h}$ 后检测 $\mathrm{CD}^{+}$记忆型 $\mathrm{T}$ 细胞亚群变化情况 B 对 $\mathrm{CD} 4+$ 效应记忆型 $\mathrm{T}$ 细胞亚群进行统计学分析结果 $(* \mathrm{p}<0.05)$
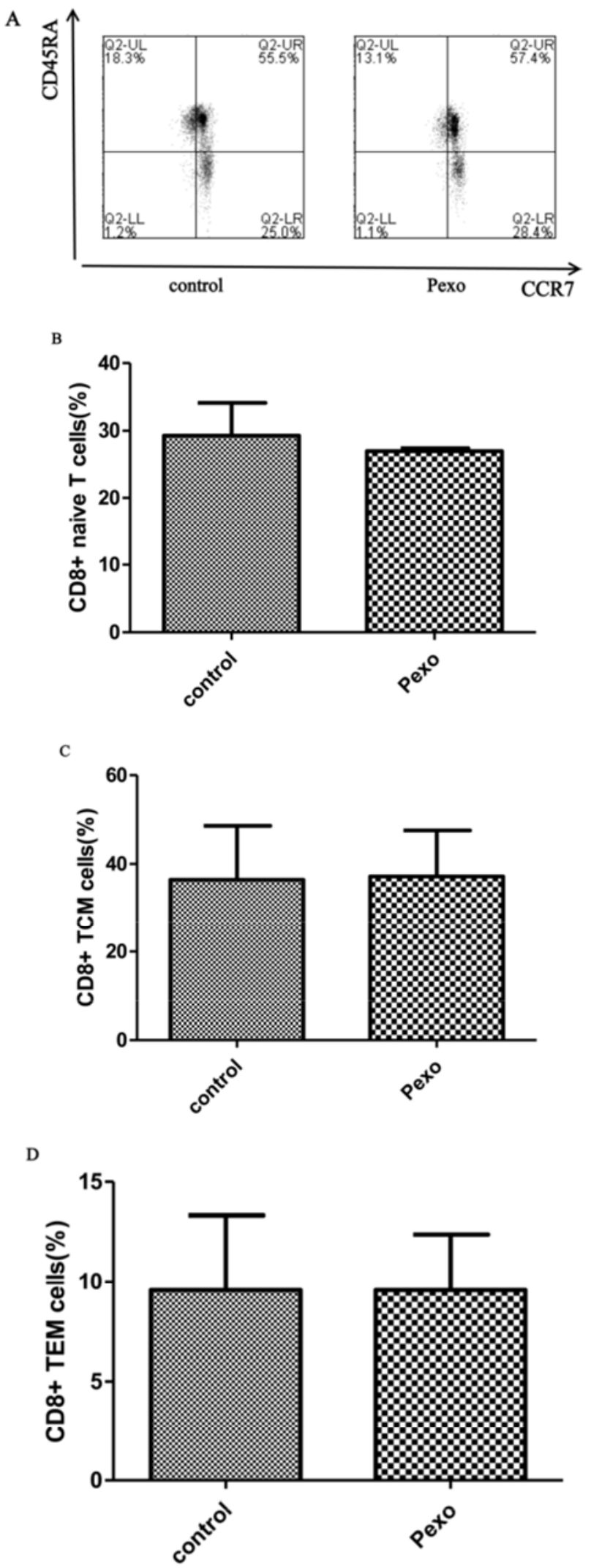

图4 Pexo对PBMCs中CD8 $8^{+}$记忆型T细胞亚群的影响。

A Pexo和PBMCs共培养 $72 \mathrm{~h}$ 后检测 $\mathrm{CD} 8^{+}$记忆型 $\mathrm{T}$ 细胞亚群变化情况 B D 分别对naive $T$ 细胞亚群、中央记忆型 $T$ 细胞以及效应记忆型 $T$ 细胞 亚群进行统计学分析结果 $(* \mathrm{p}<0.05)$ 


\subsection{Pexo对PBMCs细胞体外增殖能力的影响}

用 CFSE 对 PBMCs 染色, 流式检测在加入 Pexo后 PBMCs的增殖能力。结果发现, 加了Pexo实验组 (2.7\%)
相对于对照组 (2.1\%) 有上升的趋势, 但没有明显差异。 所以血浆外泌体并未对外周PBMCs产生明显地促进或者 抑制作用（图5）。

A
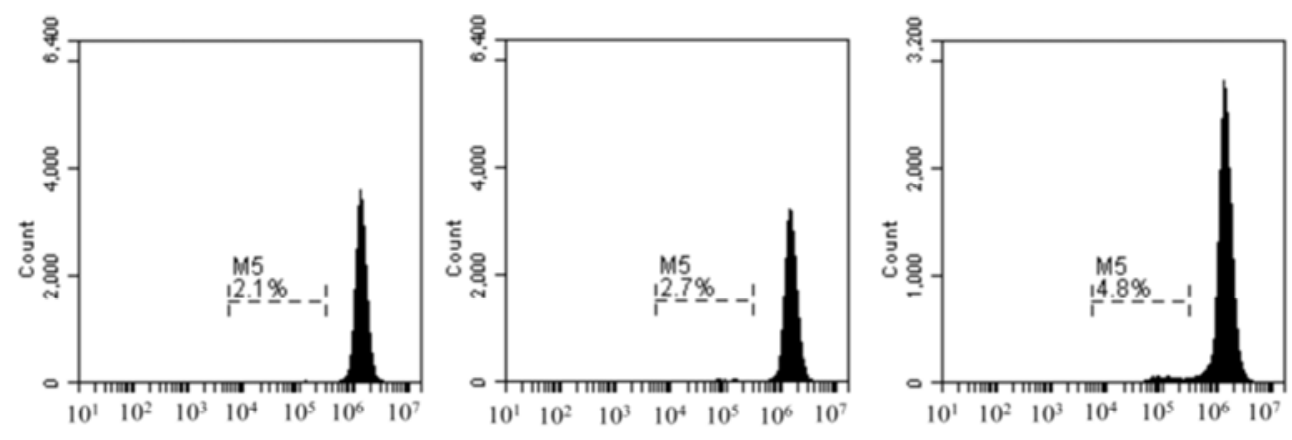

control

Pexo

$\mathrm{CD} 3 / \mathrm{CD} 28 / \mathrm{CD} 2 \quad \mathrm{CFSE}$

B

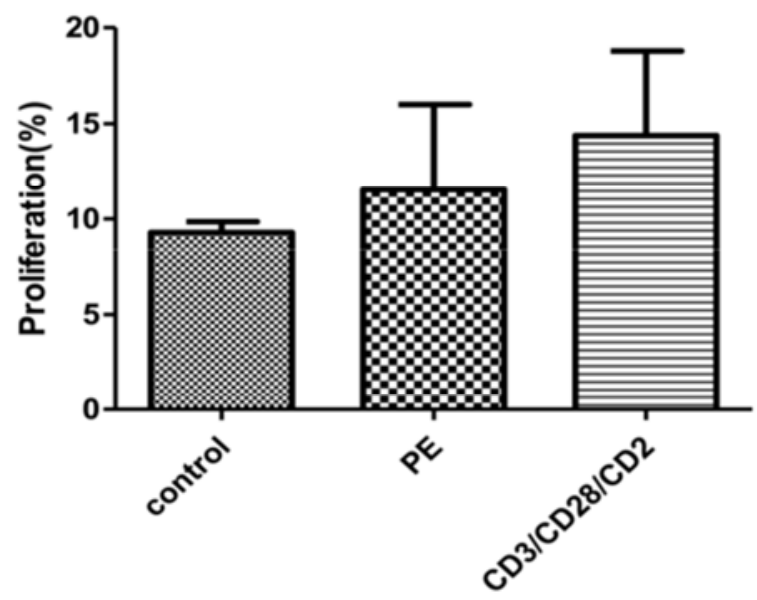

图5 Pexo对PBMCs增殖能力的影响。

A 流式细胞仪对PBMCs中细胞增殖的检测, 分别为control组（未做任何处理的PBMCs细胞） Pexo（加了 $20 u g$ 的Pexo） CD3/CD28/CD2组（25 $\mu 1 / \mathrm{ml}$ 添加） B 对重复实验进行统计学分析结果 $(* \mathrm{p}<0.05)$

\section{4. $\mathrm{CD}^{2} 5^{+}$细胞亚群增殖情况}

前面的结果看到, Pexo的加入, 并未对 T细胞各亚群 以及增殖产生明显地作用, 而 $\mathrm{CD} 25^{+}$是 $\mathrm{T}$ 细胞激活的标志,
所以此次同时对增殖细胞和 $\mathrm{CD} 25^{+}$细胞标记, 结果表明, 实验组Pexo在增殖细胞中 $\mathrm{CD} 25^{+}$表达相对于 control组无差 异（图6）。

A
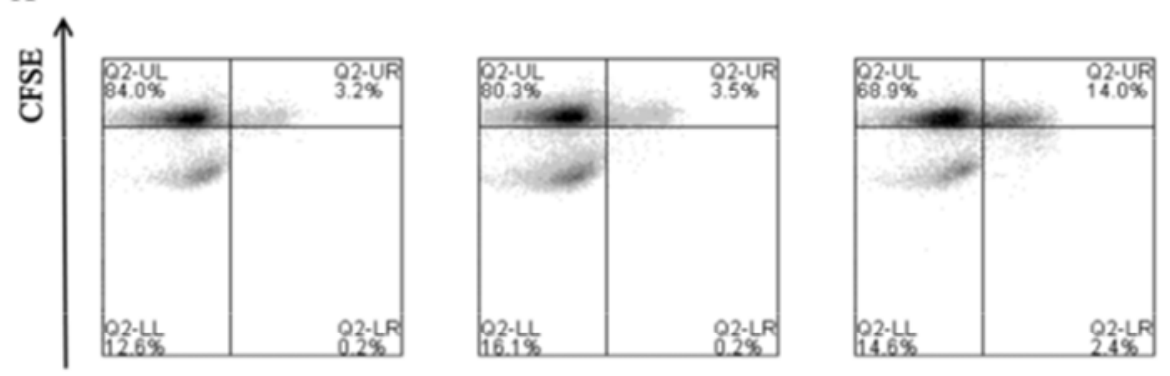

control

Pexo

$\mathrm{CD} 3 / \mathrm{CD} 28 / \mathrm{CD} 2 \mathrm{CD} 25$ 
B

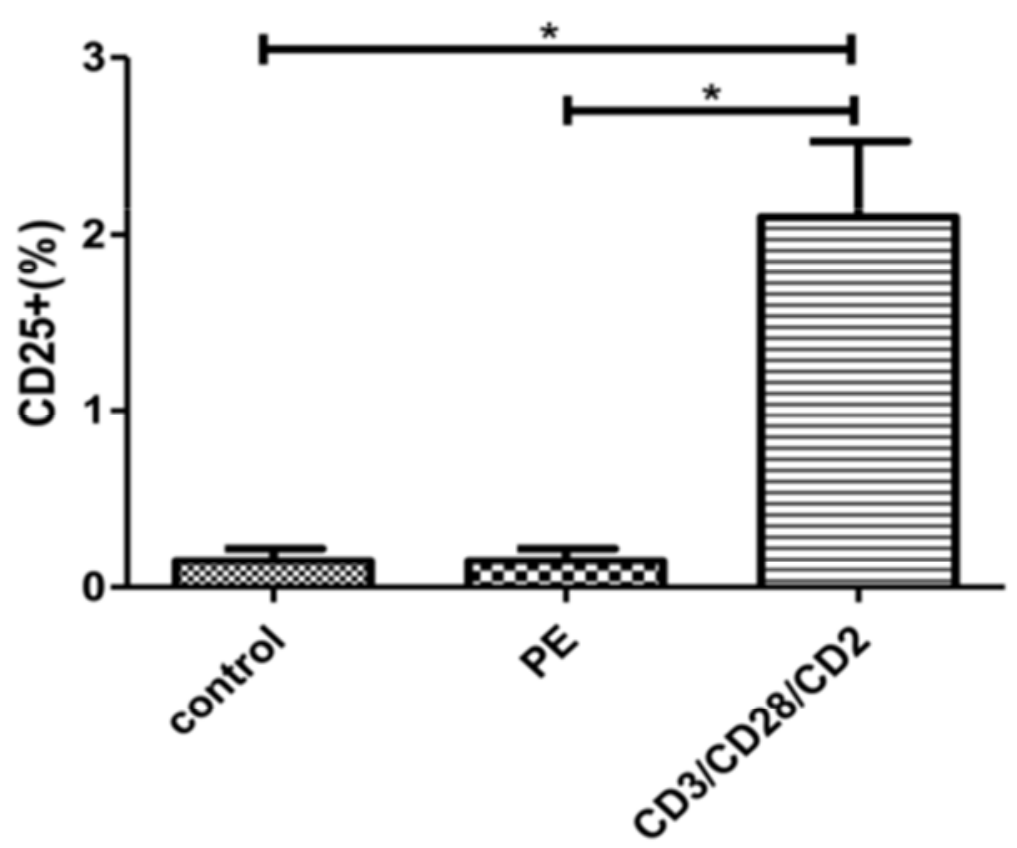

$\mathrm{A}$ 流式细胞仪检测CD25 亚群 B 对重复实验进行统计学分析结果 $(* \mathrm{p}<0.05)$

图6 对增殖细胞进行 $\mathrm{CD} 25^{+}$表达的检测。

3.5. 分别检测 $24 \mathrm{~h} 、 48 \mathrm{~h} 、 96 \mathrm{~h}$ 时PBMCs 分泌的相关免疫因

\section{子的表达变化}

前面的结果看到, 将Pexo与PBMCs共培养后, 同对照 组相比, 都未能看到血浆外泌体对 $\mathrm{T}$ 细胞本身活性及功能 亚群的改变, 因此, 我们对共培养 $24 \mathrm{~h} 、 48 \mathrm{~h}$ 及96h后PBMCs 分泌的相关细胞因子进行检测。却发现在加入Pexo后 $24 \mathrm{~h}$ 的时候就显著的下调了 IFN $-\gamma$ 和TNF- $\alpha$ 炎症因子的表达, 结果具有统计学差异 (图7)。

A

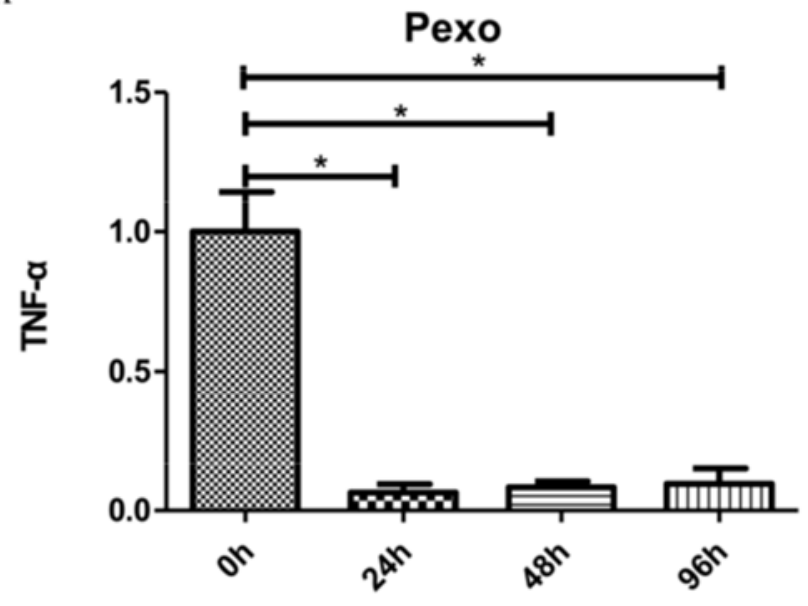

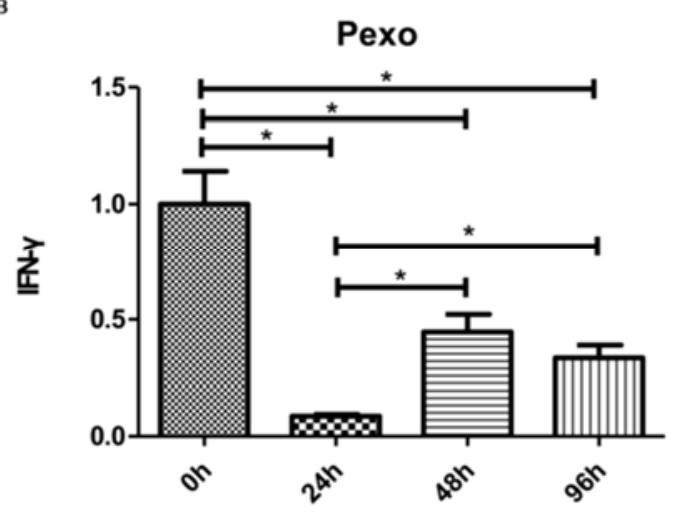

c

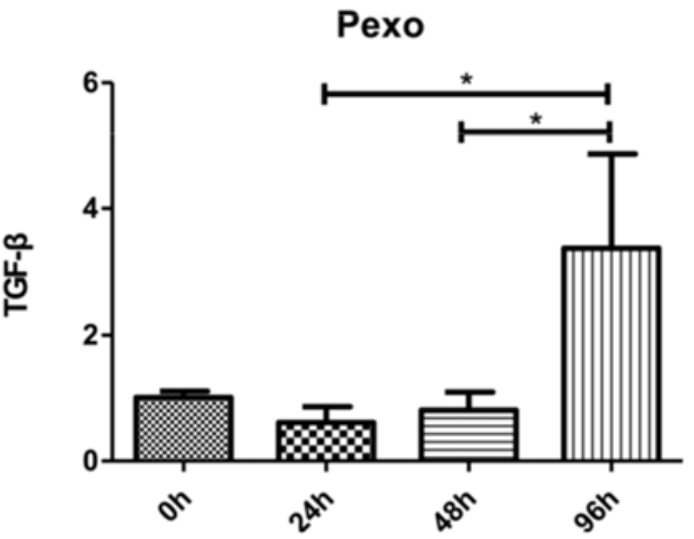


D

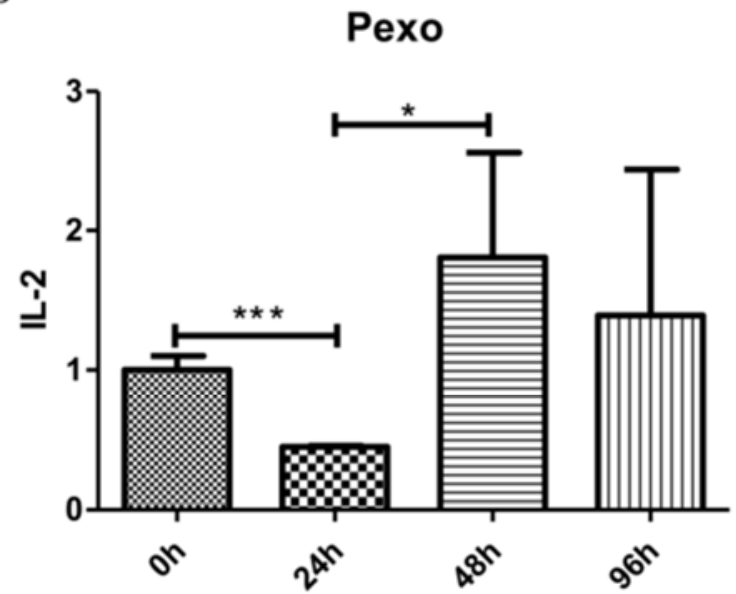

图7 24h、48h、96h时分泌的相关免疫因子的表达变化。

A-D 分别对TNF- $\alpha 、$ IFN- $\gamma 、$ TGF- $\beta$ 、IL-2表达情况进行统计学分析的结 果 $(* \mathrm{p}<0.05)$

\section{4. 结果与讨论}

近年来对脐带血的研究越来越多, 因为脐带血中免疫 细胞发育不成熟, 抗原表达和功能活性低下, 使得其在脐 血造血干细胞的成功移植及在抗肿瘤免疫中发挥了重要 作用。而外泌体是活细胞分泌的来源于晚期核内体(也称 为多囊泡体)的膜性囊泡, 存在于包括血液、唾液、尿液 和母乳等体液中 [19]。其表面一般富含脂筏及跨膜蛋白, 而其内载有蛋白质、mRNA、microRNA等生物活性分子 [20], 不同组织细胞来源exosome由于携带的蛋白质不同, 而能够发挥不同的生物学功能。目前研究较多的有肿瘤细 胞分泌的exosome, 如淋巴瘤释放的外泌体能够诱导抗原 特异性的抗淋巴瘤效应[21]; 除了肿瘤细胞, 树突状细胞 分泌的exosome能够调控抗原特异性应答等[22]。而对脐血 来源的外泌体研究较少, 尤其是脐带血血浆, 所以本文选 取脐血血浆里的外泌体为研究对象, 旨在初步探索其对人 外周淋巴细胞的免疫调节作用。

结果表明, Pexo的加入, 没有刺激 T细胞的增殖, 也未 对PBMCs细胞功能亚群产生影响, 但通过对细胞因子的检测, 却能看到加入Pexo的 $24 \mathrm{~h}$ 内, PBMCs分泌的IFN- $\gamma$ 和TNF- $\alpha$ 的 表达显著下调, 这就表明Pexo能够对炎症因子有一个较强的 调节作用, 而且是在不改变细胞本身功能亚群分布前提下。 这与报道的人血浆外泌体在体外抑制CD4+T细胞增殖, 诱导 $\mathrm{T}$ 细胞的调亡有所不同[18]。对于其中调节机制目前不是特 别清楚, 但究其来源, 本身脐血里面IFN- $\gamma$ 和TNF- $\alpha$ 的表达相 对较低[9], 而Pexo作为载体携带了脐血里面相关分子的信息 从而对PBMCs中细胞因子的表达产生了影响。有相类似的研 究表明将胎儿的DC细胞与成人淋巴细胞共培养, 也会降低 成人细胞产生TNF- $\alpha$ 的能力 $[23]$ 。

\section{5. 结论}

脐血浆来源的外泌体在体外对 $\mathrm{T}$ 细胞具有免疫调节作 用, Pexo能够显著下调炎症因子的特性有望用于相关免疫
疾病的辅助治疗, 可作为潜在的治疗由各种促炎症因子的 大剂量表达而引起的疾病, 比如恶液病等, 同时, 当前老 年人群体所占比重越来越大, 而老年人体内就处于低度炎 症的状态 [24], Pexo也许能对老年人体内的炎症状态有一 个改善作用。同时根据本实验室的另一些相关研究结果, Pexo不仅不会对细胞产生影响, 还能对细胞的培养有一定 的维持作用, 这也与相关研究运用脐带血血浆体外培养细 胞的结果相一致 [25]。

\section{致谢}

本文受国家自然科学基金基金项目（NSFC，No. 81671807 ) 资助。

\section{参考文献}

[1] Powrie F, Read S, Mottet C, et al. Control of immune pathology by regulatory $\mathrm{T}$ cells. [J]. Current Opinion in Immunology, 1998, 10(6):649-655.

[2] Shevach E M. Regulatory T cells in autoimmmunity*.[J]. Annual Review of Immunology, 2000, 18(1):423-449.

[3] Sakaguchi S. Regulatory $\mathrm{T}$ cells: key controllers of immunologic self-tolerance.[J]. Cell, 2000, 101(5):455-458.

[4] Shevach E M. CD4+ CD25+ suppressor T cells: more questions than answers.[J]. Nature Reviews Immunology, 2002, 2(6):389-400.

[5] Sakaguchi, S., N. Sakaguchi, M. Asano, M. Itoh, and M. Toda 1995. Immunologic self-tolerance maintained by activated $\mathrm{T}$ cells expressing IL-2 receptor alpha-chains (CD25). Breakdown of a single mechanism of self-tolerance causes various autoimmune diseases..[J]. Journal of Immunology, 1995, 155(3):1151-64.

[6] Cools N, Ponsaerts P, Van Tendeloo V F, et al. Regulatory T Cells and Human Disease[J]. Clinical \& Developmental Immunology, 2015, 2007(1740-2522):89195.

[7] Kondělková K, Vokurková D, Krejsek J, et al. Regulatory T cells (TREG) and their roles in immune system with respect to immunopathological disorders[J]. Acta Medica, 2010, 53(2):73.

[8] Safinia N, Sagoo P, Lechler R, et al. Adoptive regulatory T cell therapy: challenges in clinical transplantation[J]. Current Opinion in Organ Transplantation, 2010, 15(4):427.

[9] 江淑芳,史春梦.脐带血免疫学特性的研究进展 [J].免疫学杂 志, 2002,18(b06):149-152。

[10] Théry C, Zitvogel L, Amigorena S. Exosomes: composition, biogenesis and function[J]. Nature Reviews Immunology, 2002, 2(8):569.

[11] Lobb R J, Becker M, Wen S W, et al. Optimized exosome isolation protocol for cell culture supernatant and human plasma[J]. J Extracell Vesicles, 2015, 4:27031. 
[12] Sódar B W, Ágnes Kittel, Pálóczi K, et al. Low-density lipoprotein mimics blood plasma-derived exosomes and microvesicles during isolation and detection[J]. Scientific Reports, 2016, 6:24316.

[13] Goetzl E J, Boxer A, Schwartz J B, et al. Altered lysosomal proteins in neural-derived plasma exosomes in preclinical Alzheimer disease.[J]. Neurology, 2015, 85(1):40.

[14] Vicencio J M, Yellon D M, Sivaraman V, et al. Plasma exosomes protect the myocardium from ischemia-reperfusion injury.[J]. Journal of the American College of Cardiology, 2015, 65(15):1525-1536.

[15] Caby MP, Lankar D, VincendeauScherrer C, et al. Exosomal-like vesicles are present in human blood plasma.[J]. International immunology, 2005, 17(7):879.

[16] Jia R, Li J, Rui C, et al. Comparative Proteomic Profile of the Human Umbilical Cord Blood Exosomes between Normal and Preeclampsia Pregnancies with High-Resolution Mass Spectrometry.[J]. Cellular Physiology \& Biochemistry International Journal of Experimental Cellular Physiology Biochemistry \& Pharmacology, 2015, 36(6):2299-306.

[17] Yin Hu, Shan-Shan Rao, Zhen-Xing Wang, et al. Exosomes from human umbilical cord blood accelerate cutaneous wound healing through miR-21-3p-mediated promotion of angiogenesis and fibroblast function:[J]. Theranostics, 2018, $8(1): 169$.
[18] 李丹.血浆Exosomes生物学特征及血浆Exosomes对巨噬细 胞功能影响的初步研究[D].复旦大学, 2011。

[19] Colombo M, Raposo G, Théry C. Biogenesis, Secretion, and Intercellular Interactions of Exosomes and Other Extracellular Vesicles[J]. Annual Review of Cell \& Developmental Biology, 2014, 30(1):255.

[20] 李晓, 刘玲英,柴家科. 外泌体的生物学特性及临床应用的研 究进展 [J].解放军医学院学报,2015(10):1042-1044。

[21] Sun J, Yao Y, Chen L J, et al. Studies on biological properties and effects of anti-lymphoma of lymphoma cell-derived exosomes[J]. Journal of Practical Medicine, 2012.

[22] Robbins P D, Morelli A E. Regulation of immune responses by extracellular vesicles.[J]. Nature Reviews Immunology, 2014, 14(3):195.

[23] Mcgovern N, Shin A, Low G, et al. Human fetal dendritic cells promote prenatal T-cell immune suppression through arginase-2[J]. Nature, 2017, 546(7660):662.

[24] 丛秀丽,赵启明.免疫系统与机体衰老[J].中国美容医学,2017, 26(1):16-20。

[25] 丁妍,卢智勇,袁雅红,等.脐血浆与胎牛血清培养人脐带间充 质干细胞的比较 [J]. 生物医学工程学杂 志,2013(6):1279-1282。 